\title{
Tau Binds to the Distal Axon Early in Development of Polarity in a Microtubule- and Microfilament-Dependent Manner
}

\author{
Martina Kempf, ${ }^{1}$ Albrecht Clement, ${ }^{1}$ Andreas Faissner, ${ }^{1}$ Gloria Lee, ${ }^{2}$ and Roland Brandt ${ }^{1}$ \\ 1/nstitute of Neurobiology, University of Heidelberg, 69120 Heidelberg, Germany, and ${ }^{2}$ Center for Neurological Diseases, \\ Harvard Medical School, Brigham and Women's Hospital, Boston, Massachusetts 02115
}

\begin{abstract}
Microtubule-associated protein tau is localized to the axon in situ and has been implicated in the development of neuronal polarity. Here we report that tau is extracted differentially in cultured hippocampal neurons yielding an axon-specific localization under conditions that keep the integrity of the plasma membrane. The amount of bound tau increases toward the distal axon and is highest at the transition from the axonal shaft to the growth cone. This distribution is significantly different from the distribution of axonal microtubules that are most concentrated at the proximal axon. Distal binding of tau to one process appears early in development of polarity in culture and
\end{abstract}

correlates with the onset of axon formation (day 2 in culture). Binding to the distal axon requires intact microtubules and microfilaments. Distal tau binding does not stabilize microtubules selectively against drug-induced disassembly, because colchicine-induced microtubule depolymerization is highest distally. We conclude that binding of tau to the distal axon follows a complex mechanism, is an early event in the development of polarity, and reproduces the axon-specific localization of tau in situ.

Key words: tau; microtubule-associated protein; hippocampal neuron; polarity; axon; plasma membrane
A characteristic morphological feature of neurons is their polarized structure with an axonal and a somatodendritic compartment. Microtubule-associated proteins (MAPs) are thought to be involved critically in the development of this polarity. In situ, they are characteristically distributed with tau being confined to the axon and MAP2 localized to the soma and dendrites (Bernhardt and Matus, 1984; DeCamilli et al., 1984; Binder et al., 1985; Kowall and Kosik, 1987; Brion et al., 1988; Trojanowski et al., 1989).

The understanding of the properties of developing neurons mostly derives from studies of cells in vitro, in which the cellular environment can be controlled most easily and in which cells are most accessible for microscopic analysis. Of the several neuronal culture systems that have been developed, hippocampal neurons have been characterized most extensively (for review, see Banker and Waxman, 1988; Craig and Banker, 1994). Cultured hippocampal neurons acquire their characteristic form by a stereotyped sequence of developmental events, such as the formation of minor neurites and the subsequent outgrowth of one of the neurites to become an axon (Dotti et al., 1988). This development is accompanied by changes in the cytoskeletal organization (Baas et al., 1989; Sharp et al., 1995), the exclusion of polyribosomes from axons (Baas et al., 1987), and the concentration of reticular membrane in the axonal growth cone (Deitch and Banker, 1993). The onset of axon formation correlates with a polar distribution of some mole-

Received March 12, 1996; revised May 28, 1996; accepted June 19, 1996.

This work was supported by an habilitation fellowship and "Sachbeihilfe" of the Deutsche Forschungsgemeinschaft (M.K., R.B.), DFG Grant Fa 159/5-2, 3(A.C., A.F.), and National Institutes of Health Grant NS32100 (G.L.). Andreas Faissner is the recipient of a Schilling Professorship for Neuroscience. We thank Drs. Lester I. Binder and Virginia M.-Y. Lee for providing anti-tau antibody (Tau-1 and 46.1, respectively) and Alan Summerfield for excellent photographic service.

Correspondence should be addressed to Dr. Roland Brandt, Institute of Neurobiology, University of Heidelberg, Im Neuenheimer Field 364, 69120 Heidelberg, Germany.

Copyright (C) 1996 Society for Neuroscience $0270-6474 / 96 / 165583-10 \$ 05.00 / 0$ cules, such as the appearance of GAP-43 in the growth cones of neurites (Goslin and Banker, 1990) and the presence of phosphorylated forms of NF-H in a subset of neurites (Pennypacker et al., 1991). However, no compartmentalization of tau into the axon has been observed, and the segregation of the somatodendritic marker MAP2 into dendrites occurs later during development (Cáceres et al., 1984).

In cultured neurons and neural cell lines, neurite outgrowth correlates with the induction of tau (Drubin et al., 1985; Ferreira et al., 1989), and suppression of tau synthesis results in inhibition of neurite development (Hanemaaijer and Ginzburg, 1991; Shea et al., 1992; Esmaeli-Azad et al., 1994) and failure of axonal development (Cáceres and Kosik, 1990). Overexpression of tau in neuronal and non-neuronal cells results in the promotion of microtubule assembly and stability (Drubin and Kirschner, 1986; Lee and Rook, 1992; Baas et al., 1994). However, it is unclear to what extent the activities of tau on microtubules have a role in axonal development, because the C-terminal microtubule-binding domain of tau also is shared by the somatodendritic MAP2 (Goedert et al., 1991), and MAP2 also is present at the time of axonal development.

To understand the role of tau during development, we have analyzed its cellular interactions during axon formation in cultured hippocampal neurons. Our previous work has indicated that differential detergent extraction allows the immunocytochemical analysis of various tau interactions. Here we analyzed the distribution of bound tau, MAP2, and microtubules in neurons at different stages of development in culture by confocal image analysis. Drug treatments were used to characterize the involvement of different cytoskeletal elements. The results are in agreement with a model in which microtubule-bound tau interacts with a plasma membrane component that is localized specifically at the distal axon and that requires actin filaments for its subcellular localization. 


\section{MATERIALS AND METHODS}

Materials. Chemicals were purchased from Sigma (St. Louis, MO), and products for cell culture were obtained from Life Technologies (Gaithersburg, MD), unless otherwise stated.

Cell culture. Rat hippocampal neurons were prepared and grown in low-density culture according to standard procedures (Goslin and Banker, 1991) except for some modifications. In brief, hippocampi were dissected from brains of 18 -d-old rat fetuses in $37^{\circ} \mathrm{C} \mathrm{Ca-Mg-free} \mathrm{HBSS}^{-}$ supplemented with $0.6 \%$ (w/v) glucose and 7 mM HEPES, pH 7.4, treated with $0.25 \%$ trypsin for $15 \mathrm{~min}$ at $37^{\circ} \mathrm{C}$, washed three times with $\mathrm{HBSS}^{-}$, and dissociated by repeated passages through a fire-polished Pasteur pipette. The cells were plated at a density of 8000 cells $/ \mathrm{cm}^{2}$ on glass coverslips precoated with $15 \mu \mathrm{g} / \mathrm{ml}$ poly-D,L-ornithine in $0.1 \mathrm{M}$ borate buffer, $\mathrm{pH}$ 8.1, and cultivated in MEM containing the N2 supplements of Bottenstein and Sato (1979), 0.1\% (w/v) ovalbumin and $0.1 \mathrm{~mm}$ pyruvate.

Drug treatments were done as follows. To disrupt actin filaments, 0.1-0.6 $\mu \mathrm{M}$ cytochalasin B was added to hippocampal cultures $2 \mathrm{~d}$ after plating, and the incubation was continued for an additional $20 \mathrm{hr}$. To disrupt microtubules, 0.1-1.0 $\mu \mathrm{M}$ colchicine was added $3 \mathrm{~d}$ after plating, and cultures were incubated for an additional $3 \mathrm{hr}$. In all experiments, drugs were added from a $1000 \times$ stock in ethanol, and controls were performed with the same added volume of carrier only (ethanol).

Immunofluorescence microscopy. For fixation without detergent extraction, cells were incubated with $4 \%(\mathrm{w} / \mathrm{v})$ paraformaldehyde in PBS (10 $\mathrm{mm}$ phosphate buffer, $\mathrm{pH} 7.4,2.7 \mathrm{~mm} \mathrm{KCl}$, and $137 \mathrm{~mm} \mathrm{NaCl}$ ) containing $4 \%(\mathrm{w} / \mathrm{v})$ sucrose for $20 \mathrm{~min}$ at room temperature (RT). After washing with PBS, cells were incubated with $0.1 \mathrm{M}$ glycine for $20 \mathrm{~min}$ and permeabilized for $5 \mathrm{~min}$ in $0.2 \%(\mathrm{v} / \mathrm{v})$ Triton X-100 in PBS.

For saponin extractions of neurons, cells were washed with extraction buffer [80 mM PIPES/KOH, pH 6.8, 1 mM $\mathrm{MgCl}_{2}, 1 \mathrm{~mm}$ EGTA, 30\% (v/v) glycerol, and $1 \mathrm{mM}$ GTP], extracted for $30 \mathrm{sec}$ with extraction buffer containing $0.02 \%(\mathrm{w} / \mathrm{v})$ saponin, and then washed again with extraction buffer, with all steps on a heating platform set at $37^{\circ} \mathrm{C}$. Fixation was performed for $1 \mathrm{hr}$ at RT with $2 \%(\mathrm{w} / \mathrm{v})$ paraformaldehyde and $0.1 \%(\mathrm{v} / \mathrm{v})$ glutaraldehyde in extraction buffer. Cells were permeabilized with $0.1 \%$ (v/v) Triton X-100 in PBS for $30 \mathrm{~min}$, incubated for $7 \mathrm{~min}$ with $10 \mathrm{mg} / \mathrm{ml}$ $\mathrm{NaBH}_{4}$ in PBS and for $20 \mathrm{~min}$ with $0.1 \mathrm{M}$ glycine in PBS, and subsequently blocked for $1 \mathrm{hr}$ with PBS containing $1 \%(\mathrm{w} / \mathrm{v})$ BSA. Increasing the saponin concentration to $0.2 \%(\mathrm{w} / \mathrm{v})$ resulted in a decrease of staining but left the overall pattern of tau immunoreactivity unchanged. Similar staining patterns also were observed by using a previously described combined NP-40 permeabilization-fixation procedure (Lee and Rook, 1992); however, the staining was more heterogeneous, and cells with tau-positive minor neurites in addition to a strongly labeled axon were observed also.

Staining was essentially as described earlier (Lee and Rook, 1992) in PBS containing $1 \%(\mathrm{w} / \mathrm{v})$ BSA with monoclonal anti-tau (Tau-1 and 46.1), polyclonal anti-tau (7A6), affinity-purified tau polyclonal antibody (Pfeffer et al., 1983), anti-MAP2 (AP20, Boehringer Mannheim, Mannheim, Germany), anti- $\alpha$-tubulin (DM1A), and anti-acetylated tubulin antibody (6-11B-1), rhodamine-coupled donkey anti-mouse, FITCcoupled donkey anti-rabbit, and Texas Red-coupled goat anti-mouse antibody (all secondary antibodies from Jackson ImmunoResearch, West Grove, PA). Staining with primary antibodies was for $1 \mathrm{hr}$ at RT and with secondary antibodies for $30 \mathrm{~min}$. For staining of actin filaments, rhodamine-coupled phalloidin was included in the secondary antibody incubation. Coverslips were mounted in $1 \mathrm{mg} / \mathrm{ml} p$-phenylenediamine in $90 \%(\mathrm{v} / \mathrm{v})$ glycerol and $10 \%(\mathrm{v} / \mathrm{v})$ PBS. Cells were photographed with Neofluar lenses on a Zeiss Axioskop.

Confocal image analysis. Confocal image analysis used a Leica TCS 4D True confocal scanner equipped with an argon/krypton laser. Cells were imaged with a $40 \times / 1.00$ PL FLUOTAR or a $63 \times / 1.4$ PL APO oilimmersion objective and were recorded at $512 \times 512$ pixels per image. Scanning was in the standard mode (450 lines/sec). Laser scan images were optimized to achieve a linear increase in pixel intensity with increasing fluorescence. Noise suppression was achieved via accumulation in the line-averaging mode ( 8 counts/average). Tau and tubulin distributions from the same neurons were recorded by two-channel simultaneous scannings from double-stained cells. From each cell $\sim 10$ optical sections with a thickness of $\sim 200 \mathrm{~nm}$ were recorded. Summation of pixel intensities in the direction of projection was achieved by using the "extended focus" option in the three-dimensional image-processing mode. To evaluate the distribution of tau and tubulin in cellular processes, consecutive 5 - $\mu \mathrm{m}$-long (2-6 d cultures) or 10- $\mu \mathrm{m}$-long ( $8 \mathrm{~d}$ cultures) segments with a diameter slightly larger than the process were defined as random windows in the data analysis mode. To correct for background fluorescence, an average background pixel intensity was calculated for each process from three random windows close to the process and then substracted from the pixels within the segments. The total fluorescence intensity for the segments was calculated by summing the corrected intensities of the pixels comprising each segment. Process diameters were measured from the projected fluorescence images at the beginning and end of each segment, and the mean was determined. Because of the heterogeneity of axonal growth cones with respect to their size, they were not included in the quantification. To compare processes with different lengths, we calculated fluorescence intensities relative to the total process length (excluding the growth cone) in $10 \%$ steps and normalized to a maximum total fluorescence intensity of 1.0 of the respective label. For each experimental condition, three representative processes were evaluated as described above, and mean and SE were calculated.

To analyze the fluorescence distribution, we performed linear regression analysis. Normalized fluorescence distributions were plotted against the segment number (i.e., 1-10 according to the fractionation in $10 \%$ steps) and slope, and $95 \%$ confidence intervals were calculated.

\section{RESULTS \\ Distribution of total tau and bound tau in cultured hippocampal neurons}

To determine the distribution of tau in polarized neurons, lowdensity cultures of rat hippocampal neurons were analyzed. After $3 \mathrm{~d}$ in culture, hippocampal neurons contain a fast-growing axon in addition to shorter neurites, which later develop into dendrites (stage 3 neurons; Dotti et al., 1988). In paraformaldehyde-fixed and permeabilized cells, tau was present in the axon, the cellular soma, and also some minor neurites (data not shown). This is in agreement with previous reports showing either ubiquitous distribution of tau or its presence in the cell body in addition to the axon in cultured neurons (Dotti et al., 1987; Ferreira et al., 1989; Brandt et al., 1995). The tau staining patterns were very similar for different monoclonal and polyclonal tau antibodies tested [Tau-1 (Binder et al., 1985), 46.1 (Kosik et al., 1988), polyclonal 7A6 recognizing an N-terminal tau epitope, and affinity-purified tau polyclonal antibody (Pfeffer et al., 1983)]. With all antibodies tested, the staining indicated a uniform distribution of total tau in the cell body and the axon and no axon-specific tau staining.

To analyze the binding of tau in the neurons, a mild extraction protocol with saponin was used. This protocol selectively removes unbound cytosolic proteins but retains cytoskeletal membrane associations (Nakata and Hirokawa, 1987). As we have found previously, in saponin-extracted $3 \mathrm{~d}$ cultured neurons, tau was concentrated in the distal portion of the axon, whereas tau in minor neurites and most of tau in the cellular soma was extracted (Fig. 1A, left). The tau staining pattern did not change with the time of extraction (30 sec to 10 min tested; data not shown) or the antibodies used (two polyclonal and two monoclonal antibodies tested; see above). From immunoblots it was calculated that $29 \%$ $( \pm 3 \%, n=2)$ of tubulin and $74 \%( \pm 10 \%, n=2)$ of tau were extracted under the conditions used. Extraction in the presence of $1 \%$ Triton X-100 completely abolished tau staining, while microtubules remained intact (data not shown), indicating a requirement of plasma membrane components for the localization of tau.

In contrast to the distribution of tau, MAP2 was present uniformly in the axon, the cell body, and minor neurites after saponin extraction (Fig. 1A, right), which was similar to its distribution in unextracted cells (data not shown). Interestingly, at early stages of development in culture (6 d or fewer) when MAP2 was still present in most of the axons, MAP2 staining extended far into the axonal growth cones, whereas tau was concentrated at the distal axonal shaft and the growth cone neck (arrowheads, Fig. 1A). Tau was absent from the F-actin-rich distal growth cone and from 

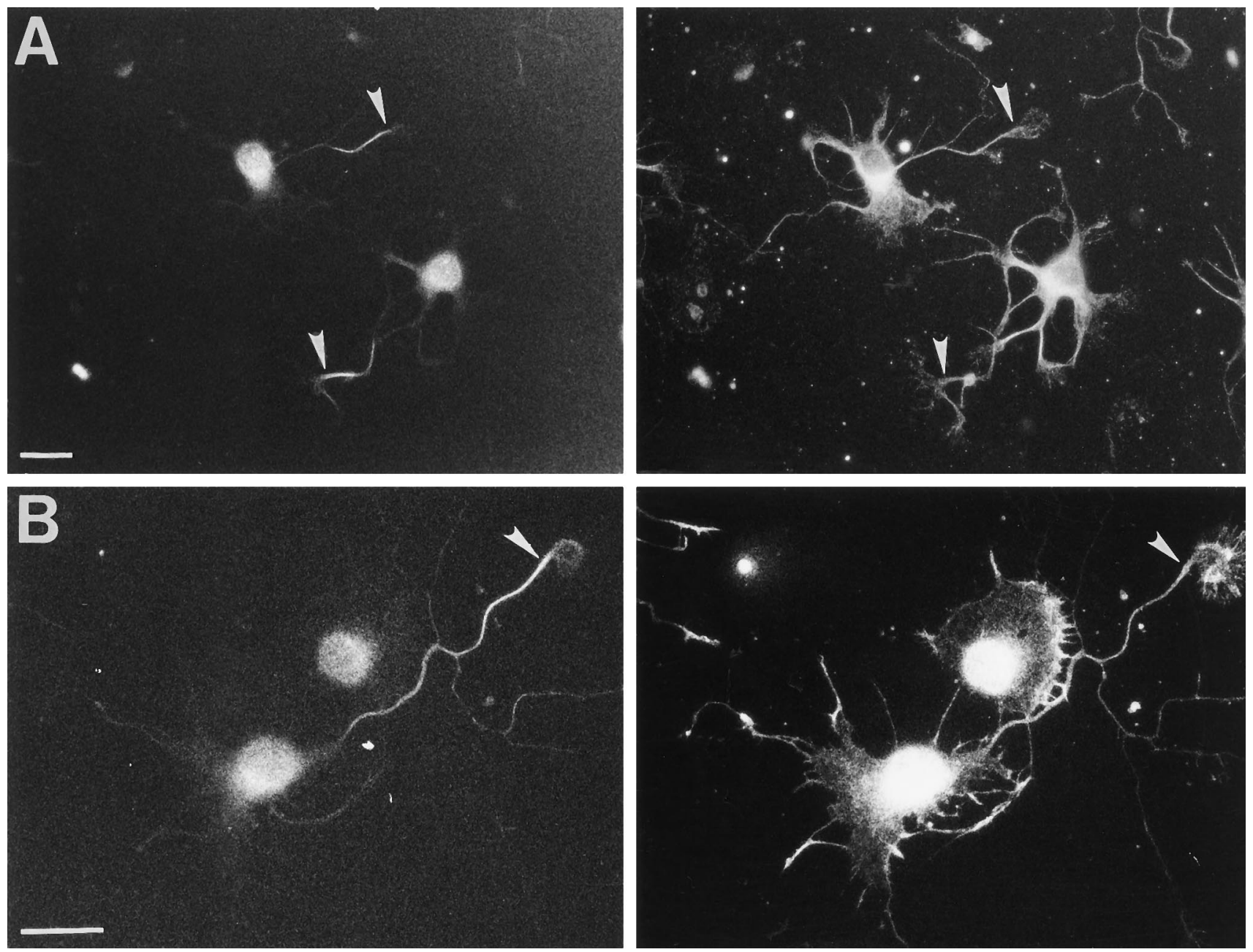

Figure 1. Tau distribution in saponin-extracted cultured hippocampal neurons. A, Double staining for tau (left) and MAP2 (right) of saponin-extracted neurons. Whereas MAP2 is present in all neurites, including the growth cones and the cellular soma, anti-tau staining is concentrated in the distal portion of the axonal shaft (arrowheads). B, Double staining for tau (left) and actin filaments (right) in saponin-extracted neurons. Note that anti-tau staining is restricted to the distal axonal shaft and the proximal growth cone (arrowheads), whereas actin filaments are prominent in the distal growth cones, including filopodia. For all experiments, cells were grown for $3 \mathrm{~d}$ in serum-free medium. Cells were extracted and fixed as described under Materials and Methods. Tau-specific staining was obtained by affinity-purified tau polyclonal and MAP2 staining by monoclonal anti-MAP2 antibody (AP20). Double staining in $A$ used FITC-coupled donkey anti-rabbit and Texas Red-coupled goat anti-mouse antibody. Actin filaments were visualized with rhodamine-coupled phalloidin in the secondary antibody reaction. Scale bar, $20 \mu \mathrm{m}$.

growth cone filopodia (Fig. $1 B$ ) and colocalized with microtubules as reported previously (Brandt et al., 1995).

To quantify the effect of saponin extraction on subcellular tau distribution, cells with tau-positive neurites after $3 \mathrm{~d}$ in culture were evaluated by visual inspection for axon-specific and distally enriched tau localization. In all, 93\% $( \pm 5 \%, n=2)$ and $90 \%$ $( \pm 2 \%, n=2)$ of extracted cells exhibited axon-specific and distally enriched tau staining, respectively, with $85 \%$ of the cells showing both. In contrast, only $5 \%( \pm 5 \%, n=2)$ of unextracted cells exhibited axon-specific staining, and in $1 \%( \pm 0 \%, n=2)$ tau staining was distally enriched.

\section{Binding of tau to the distal axon takes place early in the development of polarity in culture}

To determine the time course of the localization of tau, cultured hippocampal neurons were analyzed at different times after plating. Cultured hippocampal neurons develop by a stereotyped sequence of events, characterized by the formation of lamellipodia (stage 1), the formation of minor neurites (stage 2), and axonal outgrowth (stage 3) as early events within the first days in culture (Dotti et al., 1988). Whereas MAP2 staining in neurites was present already after $1 \mathrm{~d}$ in culture (the first time point evaluated), tau-positive processes were first observed at day 2 [Fig. $2 A$ (left), $C$ ] in $\sim 50 \%$ of the cells. The proportion of cells with tau-positive neurites constantly increased until $\sim 8 \mathrm{~d}$ in culture (the latest time analyzed), at which point almost every cell possessed one or more tau-positive neurites.

In saponin-extracted neurons, tau was enriched in the distal region of one of the neurites as soon as tau was detectable [Fig. $2 B$ (left), D], indicating that binding of tau to the distal axon is an early event in the development of polarity. In the vast majority of tau-positive cells, tau was restricted to one process and distally enriched starting from day 2 in culture; the distribution of tau was very similar during development in culture (Fig. $2 D$ ). In contrast to the localization of tau to the axon early in development, the exclusion of MAP2 from the axon took place much later, with approximately one-third of the cells still having a MAP2-positive 

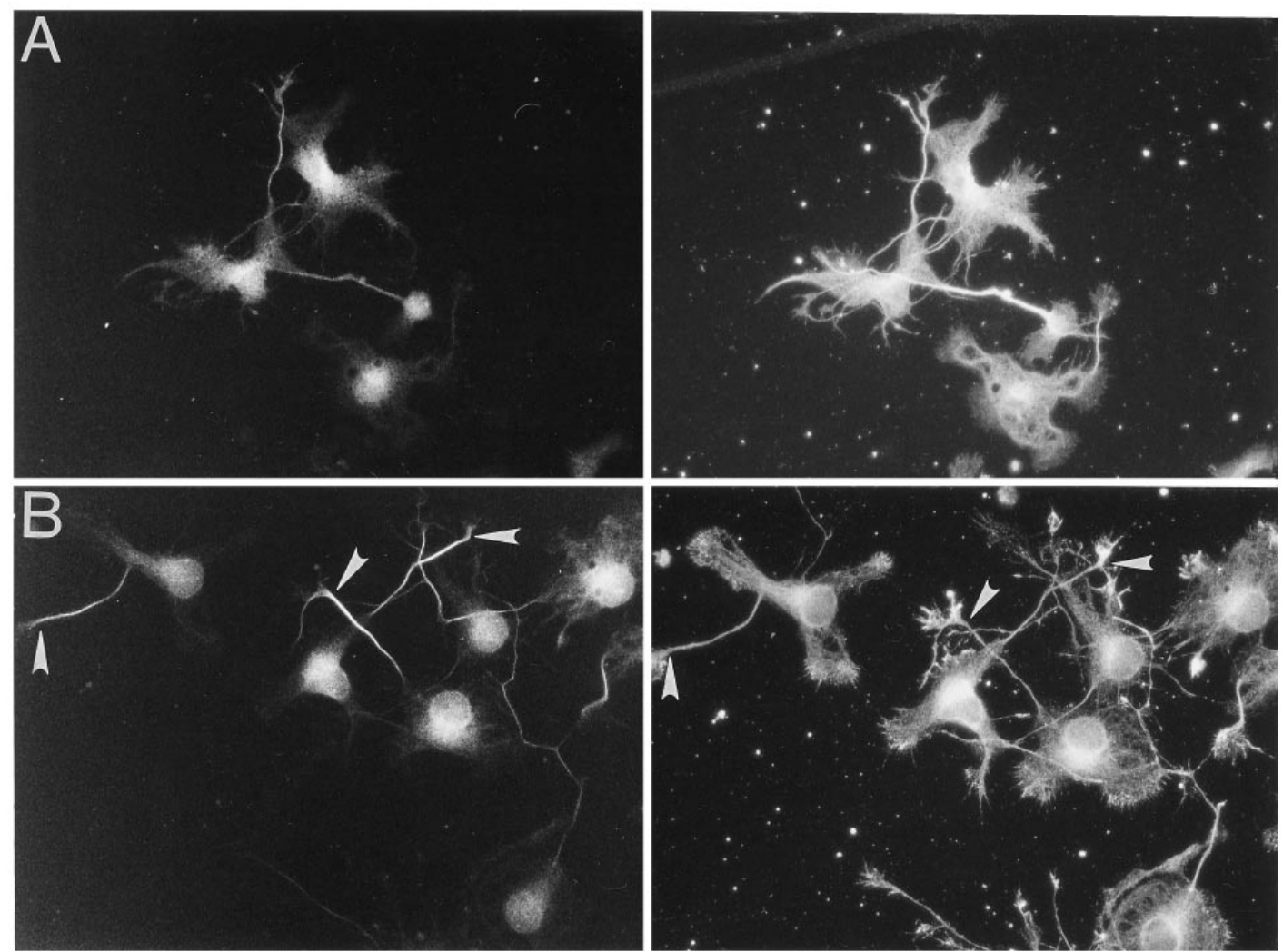

$20 \mu \mathrm{m}$
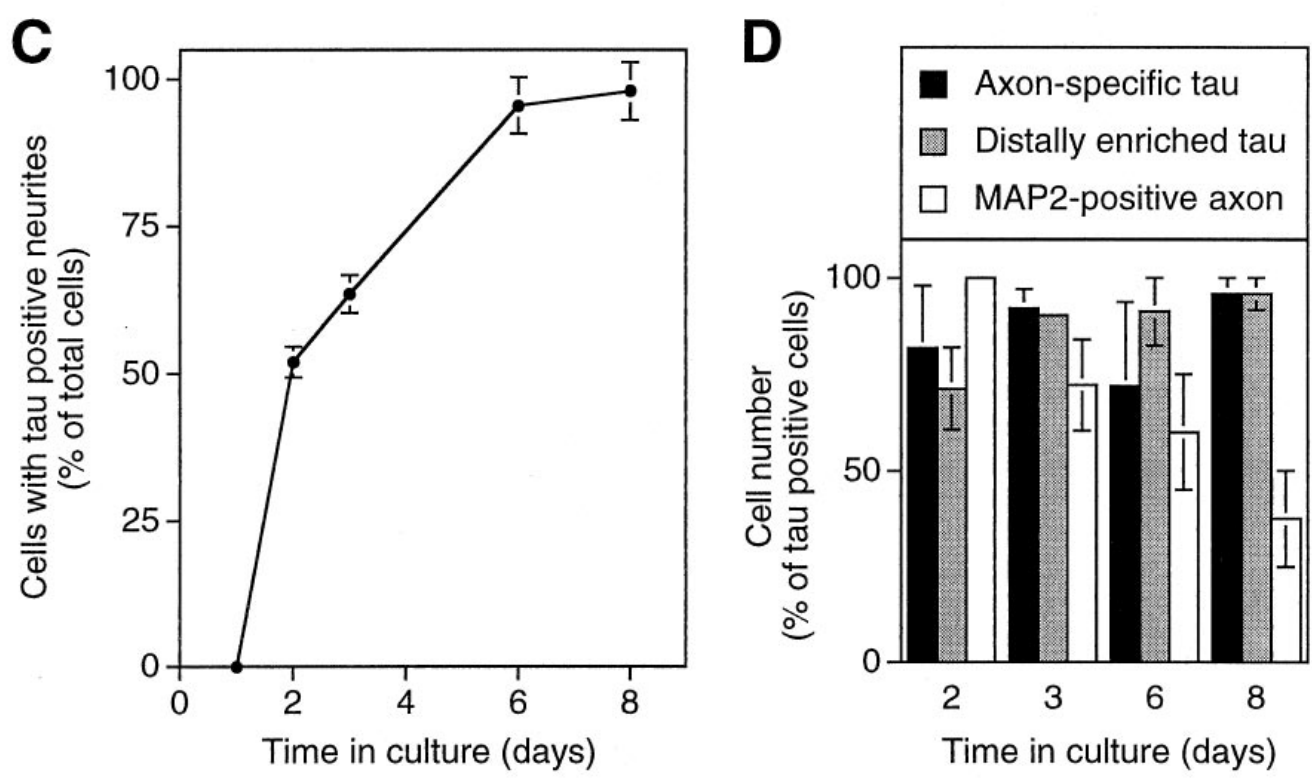

Figure 2. MAP2 and tau binding in hippocampal neurons during development in culture. $A$, Distribution of tau (left) and MAP2 (right) in unextracted hippocampal neurons after $2 \mathrm{~d}$ in culture. $B$, Double staining for tau (left) and MAP2 (right) of saponin-extracted cells after $2 \mathrm{~d}$ in culture. Note the intense MAP2 staining at the periphery of the cell in actin-rich regions (compare with Fig. $1 A$ ). In contrast, tau staining is enriched in the distal axonal shaft (arrowhead). C, Quantification of tau expression during development in culture. At times indicated, cells were fixed and stained. One hundred cells were scored for each experiment, and the proportion of cells with tau-positive neurites was determined. Mean and range from two independent experiments are shown. $D$, Quantification of cells with axon-specific and distally enriched tau staining during development in culture. At times indicated, cells were extracted, fixed, and stained. Cells with tau-positive neurites were evaluated. For 2 and 3 d cultures, between 61 and 100 cells were scored. For 6 and 8 d cultures, between 8 and 40 cells were scored; only a small portion of processes could be followed unequivocally because of the formation of an extensive neuritic meshwork. Mean and range from two independent experiments are shown. For all experiments, cells were grown for the time indicated in serum-free medium. Cells were fixed and permeabilized or extracted and fixed as described under Materials and Methods. Tau-specific staining was obtained by monoclonal antibody Tau-1 $(C)$ or affinity-purified tau polyclonal $(A, B, D)$. MAP2 staining was obtained with monoclonal anti-MAP2 antibody (AP20). Double stainings used FITC-coupled donkey anti-rabbit and rhodamine-coupled donkey anti-mouse antibody. Quantifications were performed by visual inspection of cells stained for tau and MAP2. The longest process was considered as being an "axon." 

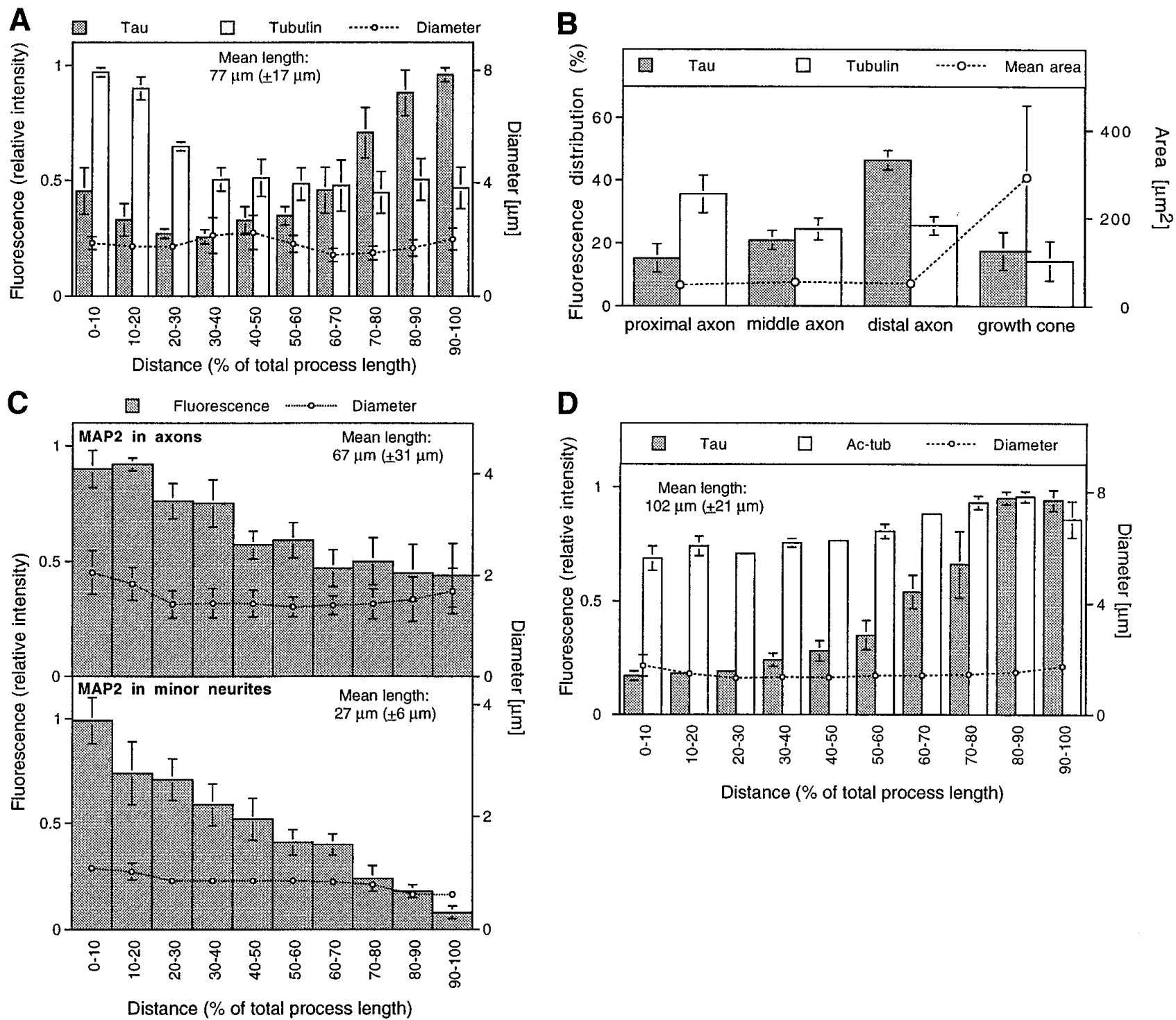

Figure 3. Distribution of bound tau, MAP2, and tubulin in processes of cultured hippocampal neurons. Distribution of tau and tubulin in axons ( $A$, $B$ ), MAP2 in axons and minor neurites $(C)$, and tau and acetylated tubulin in axons $(D)$ of cultured hippocampal neurons. Note that tau is enriched in the distal axon, which is not paralleled by a similar distribution of MAP2, tubulin, acetylated tubulin, or an increase in process diameter. For all experiments, cells were grown for $3 \mathrm{~d}$ in serum-free medium. Cells were extracted and fixed as described in Materials and Methods. Tau-specific staining was obtained by affinity-purified tau polyclonal, MAP2 staining by monoclonal anti-MAP2 (AP20), tubulin staining by monoclonal anti- $\alpha$-tubulin (DM1A), and acetylated tubulin staining by monoclonal anti-acetylated tubulin antibody (6-11B-1). Double stainings used FITC-coupled donkey anti-rabbit and Texas Red-coupled goat anti-mouse antibody. Mean and SE of the relative fluorescence intensity of three different processes are shown in each graph. For $A$ and $D$, the tau and tubulin distribution in the same axons was determined after double staining. Mean and SD of process lengths are indicated. Mean and SE of process diameters $(A, C, D)$ or area $(B)$ are shown.

axon after $8 \mathrm{~d}$ in culture (Fig. 2D). Interestingly, during early development (at days 1 and 2 in culture), MAP2 showed an intense binding at the cellular periphery at lamellipodial and filopodial regions (Fig. $2 B$, right), where it colocalized with actin filaments (data not shown). Between days 2 and 3 a switch to a microtubular staining pattern took place (compare Fig. $2 B$, right, with $1 A$, right).

The results indicate that binding of tau to one neurite at its distal end takes place early in the development of polarity in culture when axons start to emerge (day 2). Tau and MAP2 binding are not correlated temporally and spatially because the spatial specificity of MAP2, i.e., its exclusion from the axon, takes place much later in development.

\section{Binding of tau in the axon shows a proximal-to-distal increase}

To quantify the distribution of bound tau, microtubules, and MAP2 in axons, confocal image analysis was used. Saponinextracted neurons were prepared for immunofluorescence microscopy, and the distribution of label was quantified in consecutive segments along the neurites. Figure $3 A$ shows the distribution of tau and tubulin as the mean of three representative axons. The amount of bound tau in the distal axon was higher than in the proximal region, whereas the amount of tubulin showed an opposite distribution. Regression analysis confirmed that tau and tubulin distributions were significantly different, distributed with a 
slope of $0.068( \pm 0.026)$ and $-0.051( \pm 0.022)$, respectively $(95 \%$ confidence intervals are given). The axonal diameter remained almost constant, suggesting that the gradient in bound tau is not caused by the distribution of microtubules or an increase of axonal volume. Although tau is enriched in the distal third of the axonal shaft $\sim 3: 1$ relative to the proximal third, no specific enrichment as indicated by a high tau/tubulin ratio was found in the growth cone (Fig. 3B). It should, however, be noted that the growth cone sizes as reflected by the area they covered were very heterogeneous in the individual neurons. Because this heterogeneity complicated a systematic quantitative analysis of the growth cone regions, we decided to restrict our analysis to the axonal shafts. In contrast to tau, the amount of bound MAP2 decreased from the proximalto-distal axon (Fig. $3 C)$. The decrease $(-0.058 \pm 0.22)$ was not significantly different from the distribution of tubulin, suggesting that the localization of bound MAP2 is determined by its interaction with microtubules. Acetylated microtubules, which are thought to reflect more stable and less dynamic microtubule subpopulations (Webster et al., 1987), showed a slight increase toward the distal axon (Fig. 3D), but this increase was significantly lower than the increase in bound tau $(0.095 \pm 0.020$ and $0.028 \pm$ 0.010 for tau and acetylated tubulin, respectively; $95 \%$ confidence intervals are given).

To analyze the binding of tau during differentiation, the distribution of bound tau was quantified from cells at different times after plating (Fig. 4). The mean lengths of the axons increased approximately threefold from 2 to $8 \mathrm{~d}$ in culture. At all time points tau was enriched in the distal axon. Regression analysis revealed an $\sim 40 \%$ increase in the slope from 2 to $6 \mathrm{~d}$ of development. This increase, however, was not significant.

The results suggest that the mechanisms by which tau and MAP2 are bound in their respective compartment are different. Whereas MAP2 binding parallels the distribution of microtubules once polarity has been established, tau binding shows a proximalto-distal increase that is not reflected by a similar distribution of microtubules, acetylated microtubules, or MAP2.

\section{Distal tau binding depends on intact microtubules and microfilaments}

To test for the role of microtubules in the binding of tau to the distal axon, neurons were cultivated for $3 \mathrm{~d}$, followed by an incubation with the microtubule-disrupting drug colchicine (Wilson and Bryan, 1974). Cells were treated at 0.1-1 mm colchicine for $3 \mathrm{hr}$, which resulted in microtubule disruption but retained the overall cell morphology of the neurons. (Higher colchicine concentrations caused a retraction of the neurites, and lower concentrations did not seem to change microtubule distribution.) After colchicine treatment, some microtubules were still present in processes, although their staining was decreased and showed extensive fragmentation compared with control cells (Fig. 5A,B). Already, at very low colchicine concentrations, no distal tau staining was observed in extracted cells (Fig. $5 C-E$ ). Instead, tau was distributed evenly in the entire neurites, suggesting that intact microtubules are required to establish the localization of tau. Interestingly, colchicine-induced loss of microtubules preferentially occurred distally in neurites, resulting in a total loss of growth cone microtubules and a proximal-to-distal microtubule gradient (Fig. 5B). At colchicine $0.1 \mathrm{~mm}$ was already sufficient to create this effect. This indicates that the distal binding of tau does not confer stability against drug-induced disassembly of microtubules.

To analyze a possible involvement of actin filaments in the

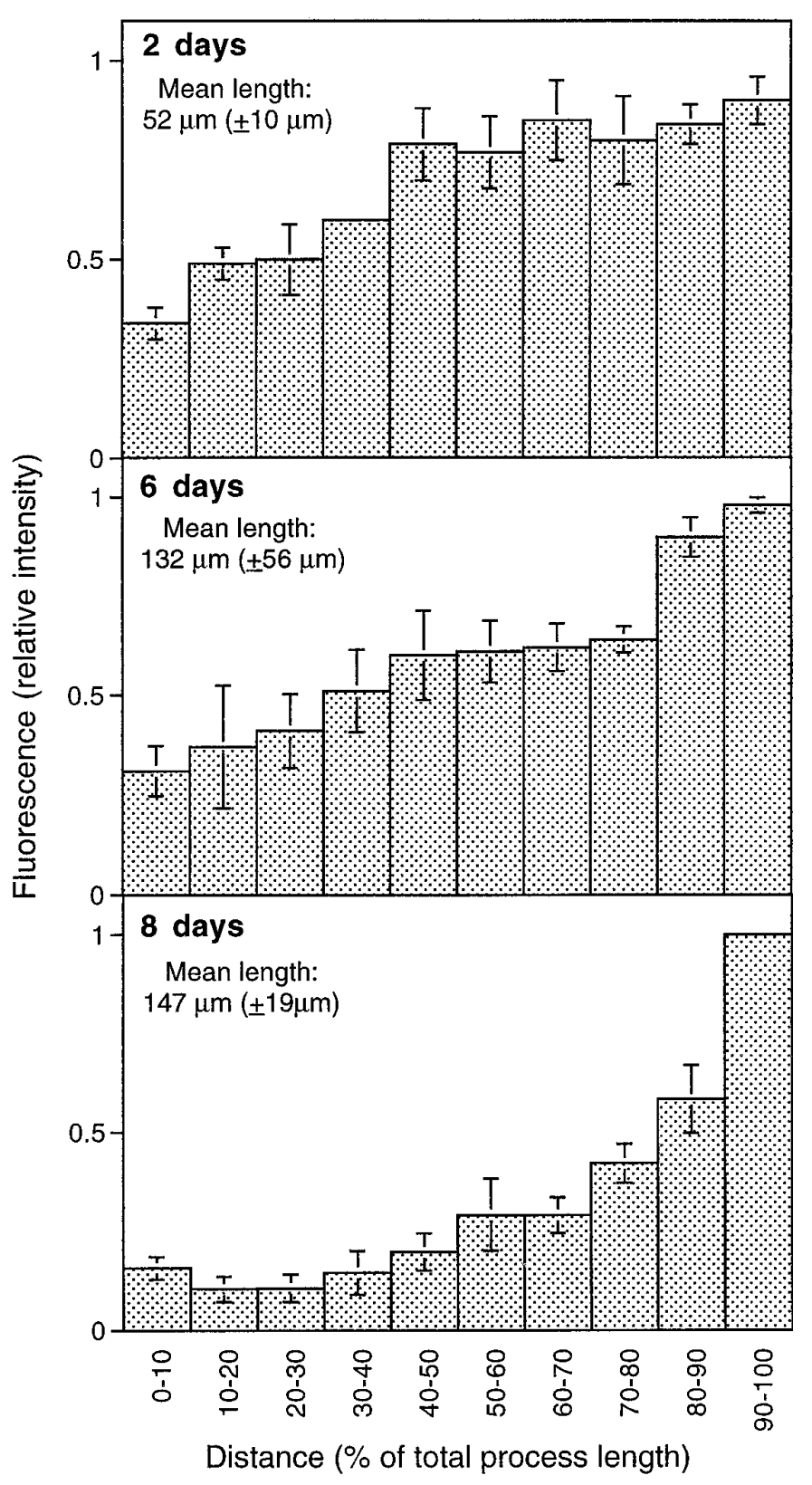

Figure 4. Time course of tau distribution in axons of cultured hippocampal neurons. Note the enrichment of tau in the distal axon at all times in culture. Cells were grown for the times indicated in serum-free medium. Cells were extracted and fixed as described in Materials and Methods. Tau-specific staining was obtained by affinity-purified tau polyclonal antibody and Texas Red-coupled goat anti-mouse secondary antibody. Mean and SE of the relative fluorescence intensity of three different processes are shown. Mean and SD of process lengths are indicated.

localization of bound tau, neurons were kept for $2 \mathrm{~d}$ in culture to allow binding of tau to the distal axon, followed by incubation with the actin filament-disrupting drug cytochalasin B (Cooper, 1987). Figure $6 A$ shows the effect of the drug on the structure of actin filaments. Control cells exhibited a strong actin filament staining in neurites and in growth cone filopodia (Fig. 6A, left), whereas cytochalasin-treated cells showed weak actin filament staining that seemed to be distributed discontinuously in neuritic shafts and collapsed into knob-like structures at the distal processes (Fig. $6 A$, right). The distribution of microtubules seemed to be unchanged 

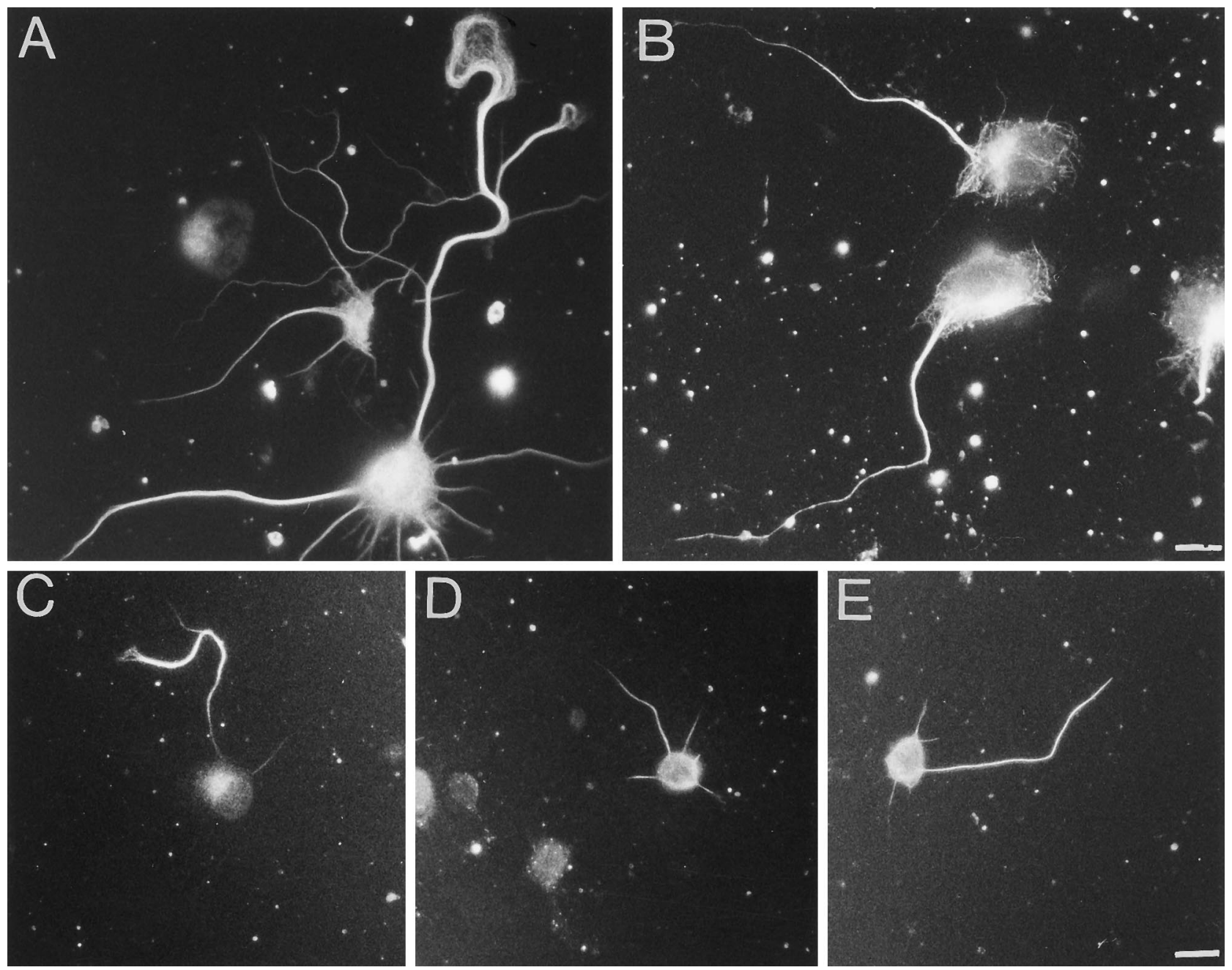

Figure 5. Effect of colchicine on tau binding in hippocampal neurons. $A$, Distribution of microtubules in hippocampal neurons, which have been treated with carrier $(0.1 \%$ ethanol) only. Note that the microtubules seem to be distributed uniformly in the axonal shaft and that they extend far into the axonal growth cone. $B$, Distribution of microtubules in colchicine-treated $(0.5 \mu \mathrm{M}, 3 \mathrm{hr})$ hippocampal neurons. Note the decrease of the microtubule concentration toward the distal axon and the complete loss of growth cone microtubules. $C-E$, Distribution of tau in control cells $(C)$ and cells treated with $0.5(D)$ and $1 \mu \mathrm{M}(E)$ colchicine. Note the redistribution of tau after colchicine treatment, resulting in a loss of the proximal-to-distal tau gradient. After $3 \mathrm{~d}$ in culture, colchicine at the indicated concentrations or, as a control, carrier only ( $0.1 \%$ final ethanol concentration) was added to the cultures. Cells were incubated for an additional $3 \mathrm{hr}$ and then extracted and fixed as described in Materials and Methods. Tau-specific staining was obtained by monoclonal anti-tau antibody (Tau-1), and microtubules were stained with monoclonal anti- $\alpha$-tubulin antibody (DM1A). Scale bars: $10 \mu \mathrm{m}(A, B) ; 20 \mu \mathrm{m}$ $(C-E)$.

in cytochalasin-treated cells indicating that, at the conditions used, cytochalasin selectively disturbs the distribution of actin filaments without inducing major morphological changes in the cells. In the majority of cytochalasin-treated cells, tau binding was no longer enriched at the distal axon but was distributed evenly in the entire axonal shaft (Fig. $6 B, C$ ). Interestingly, tau binding was still restricted to the axon, suggesting that actin-independent mechanisms operate for the axonal compartmentalization of tau. When cytochalasin-treated cells were cultured for an additional 4 $\mathrm{hr}$ in cytochalasin-free conditioned medium, a distal tau binding was observed again in some of the neurons (data not shown), indicating that the effect of cytochalasin to suppress the formation of an axonal tau gradient is reversible.

\section{DISCUSSION}

Using a differential extraction protocol designed to retain cytoskeleton plasma membrane interactions, we show in this paper that tau binds to the distal axon early in the development of neuronal polarity and remains concentrated in this region throughout maturation. This distribution contrasts with the localization of total tau, which is present ubiquitously in hippocampal neurons (our data; Dotti et al., 1987). We observed a similar binding in terminally differentiated human neurons (NT2N cells; Pleasure et al., 1992; J. Piontek and R. Brandt, unpublished observations), suggesting that binding of tau to the distal axon is a general feature of neurons that develop in culture. In the past it has proven to be difficult to find an appropriate marker for the detection of axons. Together with GAP-43, the appearance of which in growth cones correlates with the onset of axon formation (Goslin and Banker, 1990) and with the presence of phosphorylated NF-H in axons (Pennypacker et al., 1991), the staining for bound tau may be a very useful method to identify axons early in development (from day 2 in culture on).

Various mechanisms have been proposed for the segregation of tau into axons and MAP2 into dendrites. It has been proposed 

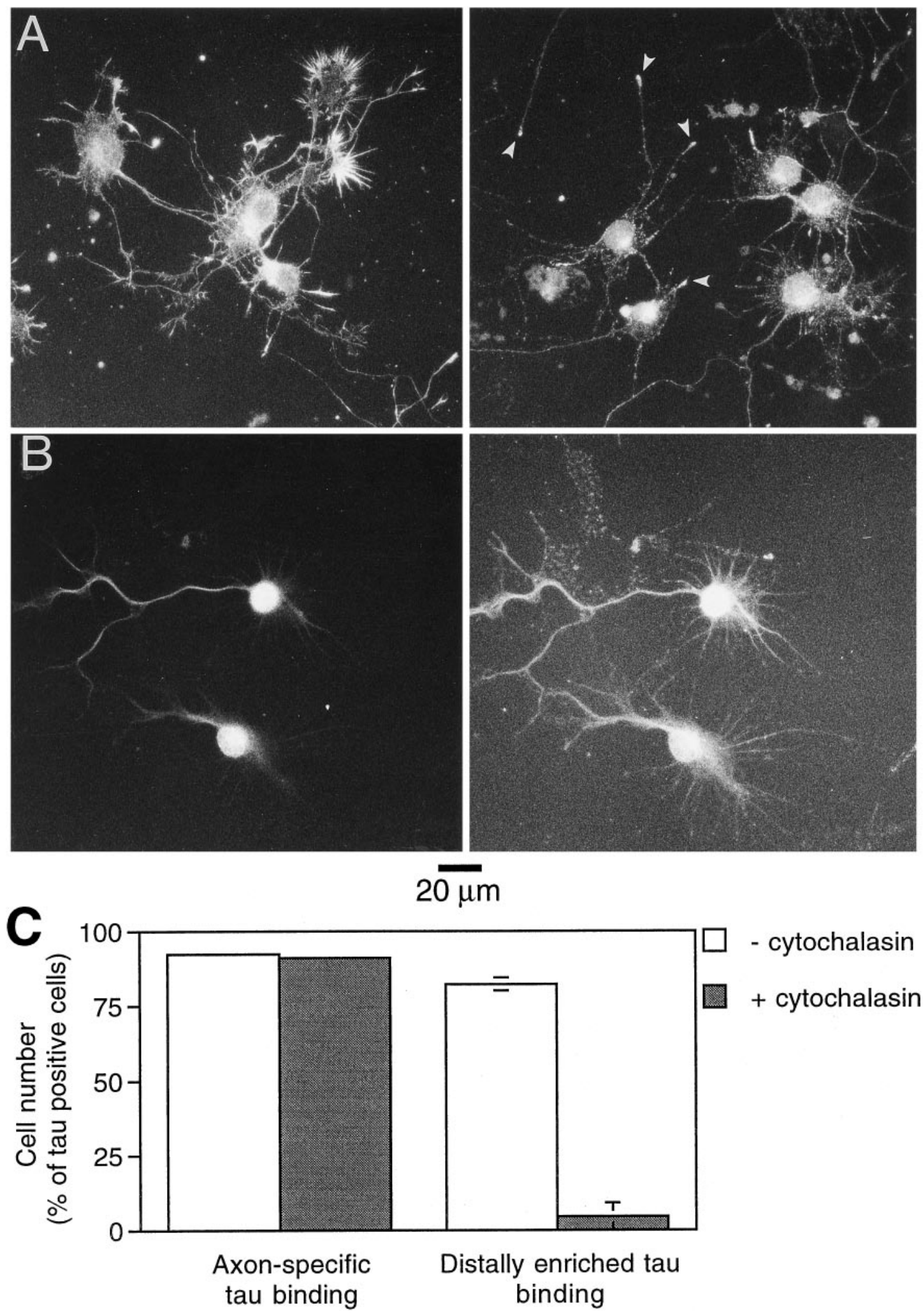

Figure 6. Effect of cytochalasin on tau binding in hippocampal neurons. $A$, Distribution of actin filaments in control cells (left) and cytochalasin-treated neurons (right). Whereas in control cells actin filaments are prominent at the periphery of the cells and in the tips of the growth cones, actin filaments seem disrupted in cytochalasin-treated cells, and knob-like structures are present in the growth cones (arrowheads). B, Distribution of tau (left) and MAP2 $($ right $)$ in cytochalasin-treated neurons. Note that tau is distributed evenly within the axon in cytochalasin-treated cells. $C$, Quantification of tau distribution in cytochalasin-treated and control cells. Mean and range from two independent experiments are shown. Between 25 and 58 tau-positive cells were scored for each experiment. After $2 \mathrm{~d}$ in culture, $0.6 \mu \mathrm{M}$ cytochalasin or, as a control, carrier only $(0.1 \%$ ethanol) was added to the cultures; the cells were incubated for an additional $20 \mathrm{hr}$ and then extracted and fixed as described in Materials and Methods. Tau-specific staining was obtained by affinity-purified tau polyclonal, MAP2 staining used monoclonal anti-MAP2 antibody (AP20), and actin filaments were stained with rhodamine-coupled phalloidin. 
that their localization is determined by mRNA localization (Garner et al., 1988; Litman et al., 1993), by locally differing protein turnover (Okabe and Hirokawa, 1989), or by locally regulated microtubule binding (Ferreira et al., 1993; Kanai and Hirokawa, 1995). However, the investigation of the mechanisms by which tau segregates into the axon has been complicated by the fact that the axon-specific localization of tau in situ has never been reproduced fully in culture in which ubiquitous or cell body staining in addition to axonal staining had been observed (Peng et al., 1986; Dotti et al., 1987; Kosik and Finch, 1987; Ferreira et al., 1989; Litman et al., 1993). Our finding that the axon-specific localization of bound tau reproduces the in situ pattern provides a tool to identify the mechanisms involved in the localization of tau. Our data indicate a complex interaction with a requirement for intact microtubules and microfilaments for establishing the binding of tau to the distal axon. At the distal axon, tau colocalizes with microtubules. Because tau clearly does not colocalize with actin filaments, cytochalasin may affect the localization of tau indirectly by affecting the interaction of the binding partner(s) of tau with microfilaments. Recently it has been shown that a microfilament-associated growth component depends on tau for its intracellular localization (DiTella et al., 1994), and it is possible that this component is part of a microtubule-tau-plasma membrane complex. Such a model is consistent with data on the functional organization of tau, which have revealed the $\mathrm{C}$-terminal domain of tau as the microtubuleinteracting unit (Lee et al., 1989; Butner and Kirschner, 1991; Brandt and Lee, 1993; Gustke et al., 1994) and the N-terminal projection domain as the part that interacts with plasma membrane components (Brandt et al., 1995).

The function of tau in the development of neuronal polarity is a matter of debate. From studies in which tau was overexpressed in non-neural or neural cells, it has been concluded that tau confers stability onto microtubules (Knops et al., 1991; Lee and Rook, 1992; Esmaeli-Azad et al., 1994), which may be a prerequisite for microtubule bundling and neurite extension. However, the microtubule-binding domain of tau and MAP2 are very similar, and both MAPs have almost identical activities toward microtubule assembly and stability (Goedert et al., 1991). This makes it unlikely that their interactions with microtubules alone are responsible for the more specific functions of the MAPs. In addition, if tau had the role of stabilizing microtubules, localizing it to the distal axon would imply a selective microtubule stabilization in this region. However, this is clearly not the case, because microtubules at the distal axon are most sensitive to drug-induced disassembly (Fig. 5). This is in agreement with previous studies in which it has been shown that the distal axon has the most dynamic microtubules (Bamburg et al., 1986). The data presented in this paper and our previous results showing that an overexpression of the $\mathrm{N}$ terminus of tau resulted in a suppression of neurite formation in PC12 cells (Brandt et al., 1995) therefore suggest that tau has a function aside from its microtubule-related activities. One may speculate that, because the addition of plasma membrane proteins at the growing axons occurs preferentially by addition at the axonal tip (Craig et al., 1995; Dai and Sheetz, 1995), tau may have a role in anchoring some of these components at the distal axon to the microtubule system. This also may help to prevent intermixing of axonal and dendritic membrane components (DeHoop and Dotti, 1993; Winckler and Poo, 1996).

As a next step in the understanding of the molecular mechanisms involved in the development of neuronal polarity, it will be important to identify the component(s) with which tau interacts at the distal axon and to analyze their function.

\section{REFERENCES}

Baas PW, Sinclair GI, Heidemann SR (1987) Role of microtubules in the cytoplasmic compartmentation of neurons. Brain Res 420:73-81.

Baas PW, MM Black, Banker GA (1989) Changes in microtubule polarity orientation during the development of hippocampal neurons in culture. J Cell Biol 109:3085-3094.

Baas PW, Pienkowski TP, Cimbalnik KA, Toyama K, Bakalis S, Ahmad FJ, Kosik KS (1994) Tau confers drug stability but not cold stability to microtubules in living cells. J Cell Sci 107:135-143.

Bamburg JR, Bray D, Chapman K (1986) Assembly of microtubules at the tip of growing axons. Nature 321:788-790.

Banker G, Waxman A (1988) Hippocampal neurons generate natural shapes in cell culture. In: Intrinsic determinants of neuronal form (Lasek R, Black MB, eds). New York: Liss.

Bernhardt R, Matus A (1984) Light and electron microscopic studies of the distribution of microtubule-associated protein 2 in rat brain: a difference between dendritic and axonal cytoskeletons. J Comp Neurol 226:203-221.

Binder LI, Frankfurter A, Rebhun LI (1985) The distribution of tau in the mammalian central nervous system. J Cell Biol 101:1371-1378.

Bottenstein JE, Sato GH (1979) Growth of a rat neuroblastoma cell line in serum-free supplemented medium. Proc Natl Acad Sci USA 76:514-519.

Brandt R, Lee G (1993) Functional organization of microtubuleassociated protein tau. Identification of regions which affect microtubule growth, nucleation, and bundle formation in vitro. J Biol Chem 268:3414-3419.

Brandt R, Léger J, Lee G (1995) Interaction of tau with the neural plasma membrane mediated by tau's amino-terminal projection domain. J Cell Biol 131:1327-1340.

Brion JP, Guilleminot J, Couchie D, Flament DJ, Nunez J (1988) Both adult and juvenile tau microtubule-associated proteins are axon specific in the developing and adult rat cerebellum. Neuroscience 25:139-146.

Butner KA, Kirschner MW (1991) Tau protein binds to microtubules through a flexible array of distributed weak sites. J Cell Biol 115:717-730.

Cáceres A, Kosik KS (1990) Inhibition of neurite polarity by tau antisense oligonucleotides in primary cerebellar neurons. Nature 343:461-463.

Cáceres A, Binder LI, Payne M, Bender P, Rebhun L, Steward O (1984) Differential subcellular localization of tubulin and the microtubuleassociated protein MAP-2 in brain tissue as revealed by immunocytochemistry with hybridoma monoclonal antibodies. J Neurosci 4:394-410.

Cooper JA (1987) Effects of cytochalasin and phalloidin on actin. J Cell Biol 105:1473-1478.

Craig AM, Banker G (1994) Neuronal polarity. Annu Rev Neurosci 17:267-310.

Craig AM, Wyborski RJ, Banker G (1995) Preferential addition of newly synthesized membrane protein at axonal growth cones. Nature 375:592-594.

Dai J, Sheetz MP (1995) Axon membrane flows from the growth cone to the cell body. Cell 83:693-701.

DeCamilli P, Miller PE, Navone F, Theurkauf WE, Vallee RB (1984) Distribution of microtubule-associated protein 2 in the nervous system of the rat studied by immunofluorescence. Neuroscience 11:817-846.

DeHoop MJ, Dotti CG (1993) Membrane traffic in polarized neurons in culture. J Cell Sci Suppl 17:85-92.

Deitch JS, Banker GA (1993) An electron microscopic analysis of hippocampal neurons developing in culture: early stages in the emergence of polarity. J Neurosci 13:4301-4315.

DiTella M, Feiguin F, Morfini G, Cáceres A (1994) Microfilamentassociated growth cone component depends upon tau for its intracellular localization. Cell Motil Cytoskeleton 29:117-130.

Dotti CG, Banker GA, Binder LI (1987) The expression and distribution of the microtubule-associated protein tau and microtubule-associated protein 2 in hippocampal neurons in the rat in situ and in cell culture. Neuroscience 23:121-130.

Dotti CG, Sullivan CA, Banker GA (1988) The establishment of polarity by hippocampal neurons in culture. J Neurosci 8:1454-1468.

Drubin DG, Kirschner MW (1986) Tau protein function in living cells. J Cell Biol 103:2739-2746.

Drubin DG, Feinstein SC, Shooter EM, Kirschner MW (1985) Nerve growth factor-induced neurite outgrowth in PC12 cells involves the coordinate induction of microtubule assembly and assembly-promoting factors. J Cell Biol 101:1799-1807. 
Esmaeli-Azad B, McCarty JH, Feinstein SC (1994) Sense and antisense transfection analysis of tau function-tau influences net microtubule assembly, neurite outgrowth, and neuritic stability. J Cell Sci 107:869-879.

Ferreira A, Busciglio J, Cáceres A (1989) Microtubule formation and neurite growth in cerebellar macroneurons which develop in vitro: evidence for the involvement of the microtubule-associated proteins, MAP-1a, HMW-MAP2, and Tau. Brain Res Dev Brain Res 49:215-228.

Ferreira A, Kincaid R, Kosik KS (1993) Calcineurin is associated with the cytoskeleton of cultured neurons and has a role in the acquisition of polarity. Mol Biol Cell 4:1225-1238.

Garner CC, Tucker RP, Matus A (1988) Selective localization of messenger RNA for cytoskeletal protein MAP2 in dendrites. Nature 336:674-677.

Goedert M, Crowther RA, Garner CC (1991) Molecular characterization of microtubule-associated proteins tau and MAP2. Trends Neurosci 14:193-199.

Goslin K, Banker G (1990) Rapid changes in the distribution of GAP-43 correlate with the expression of neuronal polarity during normal development and under experimental conditions. J Cell Biol 110:1319-1331.

Goslin K, Banker G (1991) Rat hippocampal neurons in low-density culture. In: Culturing nerve cells (Banker G, Goslin K, eds), pp 251-281. Cambridge, MA: MIT.

Gustke N, Trinczek B, Biernat J, Mandelkow E-M, Mandelkow E (1994) Domains of tau protein and interactions with microtubules. Biochemistry 33:9511-9522.

Hanemaaijer R, Ginzburg I (1991) Involvement of mature tau isoforms in the stabilization of neurites in PC12 cells. J Neurosci Res 30:163-171.

Kanai Y, Hirokawa N (1995) Sorting mechanisms of tau and MAP2 in neurons: suppressed axonal transit of MAP2 and locally regulated microtubule binding. Neuron 14:421-432.

Knops J, Kosik KS, Lee G, Pardee JD, Cohen GL, McConlogue L (1991) Overexpression of tau in a non-neuronal cell induces long cellular processes. J Cell Biol 114:725-733.

Kosik KS, Finch EA (1987) MAP2 and tau segregate into dendritic and axonal domains after the elaboration of morphologically distinct neurites: an immunocytochemical study of cultured rat cerebrum. J Neurosci 7:3142-3153.

Kosik KS, Orecchio LD, Binder L, Trojanowski JQ, Lee VM, Lee G (1988) Epitopes that span the tau molecule are shared with paired helical filaments. Neuron 1:817-825.

Kowall NW, Kosik KS (1987) Axonal disruption and aberrant localization of tau protein characterize the neuropil pathology of Alzheimer's disease. Ann Neurol 22:639-643.
Lee G, Rook SL (1992) Expression of tau protein in non-neuronal cells: microtubule binding and stabilization. J Cell Sci 102:227-237.

Lee G, Neve RL, Kosik KS (1989) The microtubule binding domain of tau protein. Neuron 2:1615-1624.

Litman P, Barg J, Rindzoonski L, Ginzburg I (1993) Subcellular localization of tau mRNA in differentiating neuronal cell culture: implications for neuronal polarity. Neuron 10:627-638.

Nakata T, Hirokawa N (1987) Cytoskeletal reorganization of human platelets after stimulation revealed by the quick-freeze deep-etch technique. J Cell Biol 105:1771-1780.

Okabe S, Hirokawa N (1989) Rapid turnover of microtubuleassociated protein MAP2 in the axon revealed by microinjection of biotinylated MAP2 into cultured neurons. Proc Natl Acad Sci USA 86:4127-4131.

Peng I, Binder LI, Black MM (1986) Biochemical and immunological analyses of cytoskeletal domains of neurons. J Cell Biol 102:252-262.

Pennypacker K, Fischer I, Levitt P (1991) Early in vitro genesis and differentiation of axons and dendrites by hippocampal neurons analyzed quantitatively with neurofilament-H and microtubule-associated protein 2 antibodies. Exp Neurol 111:25-35.

Pfeffer SR, Drubin DG, Kelley RB (1983) Identification of three coated vesicle components as $\alpha$ - and $\beta$-tubulin linked to a phosphorylated 50,000-dalton polypeptide. J Cell Biol 97:40-47.

Pleasure SJ, Page C, Lee VMY (1992) Pure, postmitotic, polarized human neurons derived from NTera 2 cells provide a system for expressing exogenous proteins in terminally differentiated neurons. J Neurosci 12:1802-1815.

Sharp DJ, Yu WQ, Baas PW (1995) Transport of dendritic microtubules establishes their nonuniform polarity. J Cell Biol 130:93-103.

Shea TB, Beermann ML, Nixon RA, Fischer I (1992) Microtubuleassociated protein tau is required for axonal neurite elaboration by neuroblastoma cells. J Neurosci Res 32:363-374.

Trojanowski JQ, Schuck T, Schmidt ML, Lee VM (1989) Distribution of tau proteins in the normal human central and peripheral nervous system. J Histochem Cytochem 37:209-215.

Webster D, Gundersem G, Bulinski J, Borisy G (1987) Differential turnover of tyrosinated and detyrosinated microtubules. Proc Natl Acad Sci USA 84:9040-9044.

Wilson L, Bryan J (1974) Biochemical and pharmacological properties of microtubules. Adv Cell Mol Biol 3:22-72.

Winckler B, Poo M (1996) No diffusion barrier at axon hillock. Nature 379:213. 